\title{
A Note on the Criteria of Optimal Solution for Replenishment Policy for Ameliorating Item with Shortages
}

\author{
Himani Dem \\ Department of Mathematics and Statistics \\ Banasthali University, P.O. Banasthali Vidyapith \\ Rajasthan-304022, India
}

\begin{abstract}
Ameliorating products are frequently seen in stock management. Considering ramp type pattern of time dependent demand for such products is a justification for the development of related inventory theory. In a recently published paper by Singh et al. (2011), such kind of problem is discussed. However, the solution given by author is incomplete because that fails to depict the case for optimal replenishment policy. In this article, the criteria for optimal solution of the considered model are found and the relevance of associated factors is discussed.
\end{abstract}

\section{Keywords}

Ramp type demand, inflation, deterioration, amelioration, shortages

\section{INTRODUCTION}

Several market scenarios can be witnessed in which demand of an item increases/decreases over a time period and after that it becomes constant such as in the case of fashionable products like a particular kind of cloth, ornaments etc. and seasonal products like particular kind of fruits, sea food etc. An initiative to use ramp type function for such demand was taken by Hill (1995). He considered increasing demand followed by steady demand and derived optimal production/purchase policy. Mandal and Pal (1998) developed the inventory model using ramp type demand for deteriorating products with shortages and only derived the solution for model starting with no shortage. Wu et al. (1999) extended the model for items which follow Weibull distribution deterioration rate. They also developed the model starting with no shortages. Wu and Ouyang (2000) derived the replenishment policies for deteriorating item having ramp type demand, considering two possible models for shortages. Wu (2001), further, continued the same trend in demand with deteriorating item following Weibull distribution and partially backlogged shortages while Giri et al. (2003) investigated the similar problem with completely backlogged shortages. Deng et al. (2007) modified the models of Mandal and Pal (1998) and $\mathrm{Wu}$ and Ouyang (2000). They also developed the algorithm to locate optimal replenishment policy, accordingly. Panda et al. (2008) considered the problem for seasonal products following ramp type demand without shortages. Skouri et al. (2009) followed the trend with Weibull deterioration rate and partial backlogging assuming the fraction of demand backlogged as a decreasing function of waiting time up to the next replenishment. Mandal (2010) also worked on the problem with similar background. Manna and
Chiang (2010) considered ramp type demand rate for deteriorating items following Weibull distribution. They assumed production rate depending on demand and allowed shortages in their model. Roy and Chaudhuri (2011) developed the model for ramp type demand with constant deterioration rate and effects of inflation and timediscounting. Recently, Singh et al. (2011) extended the model of Skouri et al. (2009) for ameliorating items under the environment of inflation and time value of money.

The model studied by this paper is identical to that of Singh et al. (2011) where they incorporated the phenomenon of amelioration and deterioration with ramp type time dependent demand and added the factors like Weibull distribution for amelioration/ deterioration rates, partial backordering and discounted cash flow (DCF), simultaneously, in their work. They used genetic algorithm for solution that could determine the optimal ordering quantity and replenishment policy. However, there were critical flaws in their solution process, rendering the resulting solution incomplete. In order to overcome this drawback, the optimal replenishment policy is established in the present work in section 4. Also, an explicit point wise study for the affects of different parameters on the optimal solution will be done which is missing in earlier paper in section 5 .

\section{ASSUMPTIONS AND NOTATIONS}

We use the same assumptions and notations as in Singh et al. (2011).

1. The inventory system involves single commodity which is ameliorating as well as deteriorating in nature.

2. The rate of amelioration is given by two parameter Weibull distribution $\gamma(\mathrm{t})=$ ght $^{\mathrm{h}-1}$ where $\mathrm{g}$ denotes the scale parameter and $\mathrm{h}$ denotes the shape parameter of deterioration rate, $\mathrm{g}<\mathrm{h}$.

3. The rate of deterioration is also given by two parameter Weibull distribution $\theta(\mathrm{t})=\alpha \beta \mathrm{t}^{\beta-1}$ where $\alpha$ denotes the scale parameter and $\beta$ denotes the shape parameter of deterioration rate, $\alpha<\beta$.

4. Demand rate follows ramp type function of time ' $t$ ' which is defined as

$\mathrm{D}(\mathrm{t})= \begin{cases}\mathrm{a}+\mathrm{bt}, & \mathrm{t}<\mu \\ \mathrm{a}+\mathrm{b} \mu, & \mathrm{t} \geq \mu\end{cases}$

where $\mathrm{a}>\mathrm{b} \geq 0$ 
5. Shortages are permitted and partially backordered at a rate $\mathrm{B}(\tau)=1 / 1+\delta(\tau), 0<\delta<1$ where $\tau$ is waiting time till next replenishment.

6. Replenishment rate is infinite.

7. Inflation and time value of money is considered and $r$ is the constant representing the difference between discount rate of time value of money and inflation rate.

8. $\quad c_{p}$ is purchasing cost per unit.

9. $c_{o}$ is ordering cost per order.

10. $\mathrm{c}_{\mathrm{h}}$ is holding cost per unit per unit time.

11. $c_{a}$ is amelioration cost per unit.

12. $\mathrm{c}_{\mathrm{d}}$ is deterioration cost per unit.

13. $\mathrm{c}_{\mathrm{s}}$ is shortage cost per unit per unit time.

14. $\mathrm{c}_{1}$ is lost sale cost per unit.

15. $\mathrm{W}$ is maximum inventory level at initial point.

16. $I_{i}(t)$ is inventory level at any time $t$ for $i^{\text {th }}$ phase, where $i=1,2,3,4$

17. $t_{1}$ is time point up to which inventory ameliorates.

18. $t_{2}$ is time at which inventory level becomes zero.

19. T is time for one replenishment cycle.

\section{MATHEMATICAL MODEL}

The problem that Singh et al. (2011) tried to solve involves three scenarios in order to represent the system.

\subsection{Case1}

When $t_{1}>\mu$, the cost function that should be minimized is

$$
\begin{gathered}
\mathrm{TC}_{1}\left(\mu, \mathrm{t}_{1}, \mathrm{t}_{2}, \mathrm{~W}\right)=\mathrm{c}_{\mathrm{o}}+\mathrm{c}_{\mathrm{p}} \mathrm{W}+\mathrm{c}_{\mathrm{h}}\left[\int_{0}^{\mu} \mathrm{I}_{1}(\mathrm{t}) \mathrm{e}^{-\mathrm{rt}} \mathrm{dt}+\int_{\mu}^{\mathrm{t}_{1}} \mathrm{I}_{2}(\mathrm{t}) \mathrm{e}^{-\mathrm{r}(\mu+\mathrm{t})} \mathrm{dt}+\int_{\mathrm{t}_{1}}^{\mathrm{t}_{2}} \mathrm{I}_{3}(\mathrm{t}) \mathrm{e}^{-\mathrm{r}\left(\mathrm{t}_{1}+\mathrm{t}\right)} \mathrm{dt}\right] \\
+\mathrm{c}_{\mathrm{a}}\left[\int_{0}^{\mu} \mathrm{ght}^{\mathrm{h}-1} \mathrm{I}_{1}(\mathrm{t}) \mathrm{e}^{-\mathrm{rt}} \mathrm{dt}+\int_{\mu}^{\mathrm{t}_{1}} \mathrm{ght}^{\mathrm{h}-1} \mathrm{I}_{2}(\mathrm{t}) \mathrm{e}^{-\mathrm{r}(\mu+\mathrm{t})} \mathrm{dt}\right] \\
+\mathrm{c}_{\mathrm{d}}\left[\int_{0}^{\mu} \alpha \beta \mathrm{t}^{\beta-1} \mathrm{I}_{1}(\mathrm{t}) \mathrm{e}^{-\mathrm{rt}} \mathrm{dt}+\int_{\mu}^{\mathrm{t}_{1}} \alpha \beta \mathrm{t}^{\beta-1} \mathrm{I}_{2}(\mathrm{t}) \mathrm{e}^{-\mathrm{r}(\mu+\mathrm{t})} \mathrm{dt}+\int_{\mathrm{t}_{1}}^{\mathrm{t}_{2}} \alpha \beta \mathrm{t}^{\beta-1} \mathrm{I}_{3}(\mathrm{t}) \mathrm{e}^{-\mathrm{r}\left(\mathrm{t}_{1}+\mathrm{t}\right)} \mathrm{dt}\right] \\
+\mathrm{c}_{\mathrm{s}} \int_{\mathrm{t}_{2}}^{\mathrm{T}}\left(-\mathrm{I}_{4}(\mathrm{t})\right) \mathrm{e}^{-\mathrm{r}\left(\mathrm{t}_{2}+\mathrm{t}\right)} \mathrm{dt}+\mathrm{c}_{1} \int_{\mathrm{t}_{2}}^{\mathrm{T}}\left(1-\frac{1}{1+\delta(\mathrm{T}-\mathrm{t})}\right)(\mathrm{a}+\mathrm{b} \mu) \mathrm{e}^{-\mathrm{r}\left(\mathrm{t}_{2}+\mathrm{t}\right)} \mathrm{dt}
\end{gathered}
$$

subject to $\mu<\mathrm{t}_{1}<\mathrm{t}_{2}<\mathrm{T}$,

$$
\mathrm{e}^{-\alpha \mathrm{t}_{1}{ }^{\beta}}\left[\begin{array}{l}
(\mathrm{a}+\mathrm{b} \mu)\left(-\mathrm{t}_{1}-\frac{\alpha \mathrm{t}_{1}^{\beta+1}}{\beta+1}+\mathrm{t}_{2}+\frac{\alpha \mathrm{t}_{2}^{\beta+1}}{\beta+1}\right) \\
-\mathrm{e}^{\mathrm{gt} \mathrm{t}_{1}^{\mathrm{h}}}\left\{-(\mathrm{a}+\mathrm{b} \mu)\left(\mathrm{t}_{1}+\frac{\alpha \mathrm{t}_{1}^{\beta+1}}{\beta+1}-\frac{\mathrm{gt}_{1}^{\mathrm{h}+1}}{\mathrm{~h}+1}\right)\right. \\
\left.+\mathrm{b}\left(\frac{\mu^{2}}{2}+\frac{\alpha \mu^{\beta+2}}{(\beta+1)(\beta+2)}-\frac{\mathrm{g} \mu^{\mathrm{h}+2}}{(\mathrm{~h}+1)(\mathrm{h}+2)}\right)+\mathrm{W}\right\}
\end{array}\right]=0
$$

and

$\mu, t_{1}, t_{2}, W \geq 0$

\subsection{Case2}

When $\mathrm{t}_{1}<\mu$, the cost function that should be minimized is

$$
\begin{aligned}
& \mathrm{TC}_{2}\left(\mu, \mathrm{t}_{1}, \mathrm{t}_{2}, \mathrm{~W}\right)=\mathrm{c}_{\mathrm{o}}+\mathrm{c}_{\mathrm{p}} \mathrm{W}+\mathrm{c}_{\mathrm{h}}\left[\int_{0}^{\mathrm{t}_{1}} \mathrm{I}_{1}(\mathrm{t}) \mathrm{e}^{-\mathrm{tt}} \mathrm{dt}+\int_{\mathrm{t}_{1}}^{\mu} \mathrm{I}_{2}(\mathrm{t}) \mathrm{e}^{-\mathrm{r}\left(\mathrm{t}_{1}+\mathrm{t}\right)} \mathrm{dt}\right. \\
& \left.+\int_{\mu}^{\mathrm{t}_{2}} \mathrm{I}_{3}(\mathrm{t}) \mathrm{e}^{-\mathrm{r}(\mu+\mathrm{t})} \mathrm{dt}\right]+\mathrm{c}_{\mathrm{a}} \int_{0}^{\mathrm{t}_{1}} \mathrm{ght}^{\mathrm{h}-1} \mathrm{I}_{1}(\mathrm{t}) \mathrm{e}^{-\mathrm{rt}} \mathrm{dt} \\
& +\mathrm{c}_{\mathrm{d}}\left[\int_{0}^{\mathrm{t}_{1}} \alpha \beta \mathrm{t}^{\beta-1} \mathrm{I}_{1}(\mathrm{t}) \mathrm{e}^{-\mathrm{rt}} \mathrm{dt}+\int_{\mathrm{t}_{1}}^{\mu} \alpha \beta \mathrm{t}^{\beta-1} \mathrm{I}_{2}(\mathrm{t}) \mathrm{e}^{-\mathrm{r}\left(\mathrm{t}_{1}+\mathrm{t}\right)} \mathrm{dt}\right. \\
& \left.+\int_{\mu}^{\mathrm{t}_{2}} \alpha \beta \mathrm{t}^{\beta-1} \mathrm{I}_{3}(\mathrm{t}) \mathrm{e}^{-\mathrm{r}(\mu+\mathrm{t})} \mathrm{dt}\right]+\mathrm{c}_{\mathrm{s}} \int_{\mathrm{t}_{2}}^{\mathrm{T}}\left(-\mathrm{I}_{4}(\mathrm{t})\right) \mathrm{e}^{-\mathrm{r}\left(\mathrm{t}_{2}+\mathrm{t}\right)} \mathrm{dt} \\
& +\mathrm{c}_{1} \int_{\mathrm{t}_{2}}^{\mathrm{T}}\left(1-\frac{1}{1+\delta(\mathrm{T}-\mathrm{t})}\right)(\mathrm{a}+\mathrm{b} \mu) \mathrm{e}^{-\mathrm{r}\left(\mathrm{t}_{2}+\mathrm{t}\right)} \mathrm{dt}
\end{aligned}
$$

subject to $\mathrm{t}_{1}<\mu<\mathrm{t}_{2}<\mathrm{T}$,

$$
\begin{aligned}
& -(a+b \mu)\left(t_{2}+\frac{\alpha t_{2}^{\beta+1}}{\beta+1}\right)+\frac{b \mu^{2}}{2} \\
& -e^{g t_{1}{ }^{h}}\left\{-a\left(t_{1}+\frac{\alpha t_{1}^{\beta+1}}{\beta+1}-\frac{g t_{1}^{h+1}}{h+1}\right)+b\left(\frac{t_{1}^{2}}{2}+\frac{\alpha t_{1}^{\beta+2}}{\beta+2}-\frac{g t_{1}^{h+2}}{h+2}\right)-W\right\} \\
& +\left(a+b t_{1}\right) \frac{\alpha t_{1}^{\beta+1}}{\beta+1}-\frac{b \alpha t_{1}^{\beta+2}}{(\beta+1)(\beta+2)}+a t_{1}+\frac{b t_{1}^{2}}{2}=0
\end{aligned}
$$

and

$\mu, t_{1}, t_{2}, W \geq 0$

\subsection{Case3}

When $t_{1}<t_{2}<\mu$, the cost function that should be minimized is

$$
\begin{aligned}
& \mathrm{TC}_{3}\left(\mu, \mathrm{t}_{1}, \mathrm{t}_{2}, \mathrm{~W}\right)=\mathrm{c}_{\mathrm{o}}+\mathrm{c}_{\mathrm{p}} \mathrm{W}+\mathrm{c}_{\mathrm{h}}\left[\int_{0}^{\mathrm{t}_{1}} \mathrm{I}_{1}(\mathrm{t}) \mathrm{e}^{-\mathrm{rt}} \mathrm{dt}+\int_{\mathrm{t}_{1}}^{\mathrm{t}_{2}} \mathrm{I}_{2}(\mathrm{t}) \mathrm{e}^{-\mathrm{r}\left(\mathrm{t}_{1}+\mathrm{t}\right)} \mathrm{dt}\right] \\
& +c_{a} \int_{0}^{t_{1}} \operatorname{ght}^{\mathrm{h}-1} \mathrm{I}_{1}(\mathrm{t}) \mathrm{e}^{-\mathrm{rt}} \mathrm{dt}+\mathrm{c}_{\mathrm{d}}\left[\int_{0}^{\mathrm{t}_{1}} \alpha \beta \mathrm{t}^{\beta-1} I_{1}(\mathrm{t}) \mathrm{e}^{-\mathrm{rt}} d t+\int_{\mathrm{t}_{1}}^{\mathrm{t}_{2}} \alpha \beta \mathrm{t}^{\beta-1} I_{2}(\mathrm{t}) \mathrm{e}^{-\mathrm{r}\left(\mathrm{t}_{1}+\mathrm{t}\right)} \mathrm{dt}\right] \\
& +\mathrm{c}_{\mathrm{s}}\left[\int_{\mathrm{t}_{2}}^{\mu}\left(-\mathrm{I}_{3}(\mathrm{t})\right) \mathrm{e}^{-\mathrm{r}(\mu+\mathrm{t})} \mathrm{dt}+\int_{\mathrm{t}_{2}}^{\mathrm{T}}\left(-\mathrm{I}_{4}(\mathrm{t})\right) \mathrm{e}^{-\mathrm{r}\left(\mathrm{t}_{2}+\mathrm{t}\right)} \mathrm{dt}\right] \\
& +c_{1}\left[\int_{t_{2}}^{\mu}\left(1-\frac{1}{1+\delta(T-t)}\right)(a+b t) e^{-r\left(t_{2}+t\right)} d t+\int_{\mu}^{T}\left(1-\frac{1}{1+\delta(T-t)}\right)(a+b \mu) e^{-r(\mu+t)} d t\right]
\end{aligned}
$$

subject to $\mathrm{t}_{1}<\mathrm{t}_{2}<\mu<\mathrm{T}$,

$$
\begin{aligned}
& \mathrm{e}^{\mathrm{gt}_{1}{ }^{\mathrm{h}}}\left\{-\mathrm{a}\left(\mathrm{t}_{1}+\frac{\alpha \mathrm{t}_{1}{ }^{\beta+1}}{\beta+1}-\frac{\mathrm{gt}_{1}^{\mathrm{h}+1}}{\mathrm{~h}+1}\right)-\mathrm{b}\left(\frac{\mathrm{t}_{1}^{2}}{2}+\frac{\alpha \mathrm{t}_{1}^{\beta+2}}{\beta+2}-\frac{\mathrm{gt}_{1}^{\mathrm{h}+2}}{\mathrm{~h}+2}\right)+\mathrm{W}\right\} \\
& +\left(\mathrm{a}+\mathrm{bt} \mathrm{t}_{1}\right) \frac{\alpha \mathrm{t}_{1}^{\beta+1}}{\beta+1}-\frac{\mathrm{b \alpha t_{1 }}{ }^{\beta+2}}{(\beta+1)(\beta+2)} \\
& +\mathrm{at}_{1}+\frac{\mathrm{bt}_{1}{ }^{2}}{2}-\left(a+b \mathrm{t}_{2}\right) \frac{\alpha \mathrm{t}_{2}^{\beta+1}}{\beta+1}+\frac{\mathrm{btt}_{2}{ }^{\beta+2}}{(\beta+1)(\beta+2)}-\mathrm{at}_{2}-\frac{\mathrm{bt}_{2}{ }^{2}}{2}=0
\end{aligned}
$$

and

$\mu, \mathrm{t}_{1}, \mathrm{t}_{2}, \mathrm{~W} \geq 0$ 


\section{OPTIMAL REPLENISHMENT}

\section{POLICY}

As per the above description of problem, three different possible cases need to be studied in order to justify the proposed model. The optimal policy will be any of the cases that give the least value of the total relevant cost. In order to achieve the goal, we can put the above derived total cost functions with their constraints for each case as a constrained nonlinear optimization problem as given below:

Case 1: $\mathrm{P}_{1}=\min \mathrm{TC}_{1}\left(\mu, \mathrm{t}_{1}, \mathrm{t}_{2}, \mathrm{~W}\right)$ subject to $\mu<\mathrm{t}_{1}<\mathrm{t}_{2}<\mathrm{T}$

Case 2: $P_{2}=\min \mathrm{TC}_{2}\left(\mu, \mathrm{t}_{1}, \mathrm{t}_{2}, \mathrm{~W}\right)$ subject to $\mathrm{t}_{1}<\mu<\mathrm{t}_{2}<\mathrm{T}$

Case 3: $\mathrm{P}_{3}=\min \mathrm{TC}_{3}\left(\mu, \mathrm{t}_{1}, \mathrm{t}_{2}, \mathrm{~W}\right)$ subject to $\mathrm{t}_{1}<\mathrm{t}_{2}<\mu<\mathrm{T}$

The equation of each $\mathrm{TC}_{\mathrm{i}}\left(\mu, \mathrm{t}_{1}, \mathrm{t}_{2}, \mathrm{~W}\right)$ and its constraint, for $i=1,2,3$, is obtained from the characteristic of the system as described in sections $3.1,3.2$ and 3.3 respectively. This problem can be solved by using an appropriate method. After computing optimal values of $\mu, \mathrm{t}_{1}, \mathrm{t}_{2}, \mathrm{~W}$ and $\mathrm{TC}_{\mathrm{i}}$, denoted by $\mu^{*}, \mathrm{t}_{1}{ }^{*}, \mathrm{t}_{2}{ }^{*}, \mathrm{~W}^{*}$ and $\mathrm{TC}_{\mathrm{i}}{ }^{*}$ respectively for $\mathrm{i}=1,2,3$, find $\min \left\{\mathrm{TC}_{\mathrm{i}}{ }^{*}\left(\mu^{*}, \mathrm{t}_{1}{ }^{*}, \mathrm{t}_{2}{ }^{*}, \mathrm{~W}^{*}\right)\right\}$. If the optimal strategy is case 1 , then the optimal ordering quantity, denoted by $\mathrm{Q}^{*}$ is given by $\mathrm{W}^{*}+\frac{\left(\mathrm{a}+\mathrm{b} \mu^{*}\right)}{\delta} \log \left\{1+\delta\left(\mathrm{T}-\mathrm{t}_{2}{ }^{*}\right)\right\}$, where $\mathrm{W}^{*}, \mu^{*}, \mathrm{t}_{2}{ }^{*}$ are optimal values of $\mathrm{W}, \mu, \mathrm{t}_{2}$, respectively for case 1 .

If the optimal strategy is case 2 , then the optimal ordering quantity, denoted by $Q^{*}$ is given by $\mathrm{W}^{*}+\frac{\left(\mathrm{a}+\mathrm{b} \mu^{*}\right)}{\delta} \log \left\{1+\delta\left(\mathrm{T}-\mathrm{t}_{2}{ }^{*}\right)\right\}$, where $\mathrm{W}^{*}, \mu^{*}, \mathrm{t}_{2}{ }^{*}$ are optimal values of $\mathrm{W}, \mu, \mathrm{t}_{2}$, respectively for case 2 .

If the optimal strategy is case 2, then the optimal ordering quantity, denoted by $\mathrm{Q}^{*}$ is given by $\mathrm{W}^{*}-\frac{\left(\mathrm{a}+\mathrm{b} \mu^{*}\right)}{\delta} \log \left\{1+\delta\left(\mathrm{T}-\mu^{*}\right)\right\}$ $+\frac{1}{\delta}\left\{\mathrm{a}+\mathrm{b}\left(\mathrm{T}+\frac{1}{\delta}\right)\right\} \log \left(\frac{1+\delta\left(\mathrm{T}-\mu^{*}\right)}{1+\delta\left(\mathrm{T}-\mathrm{t}_{2}{ }^{*}\right)}\right)+\frac{\mathrm{b}}{\delta^{2}}\left(\mu^{*}-\mathrm{t}_{2}{ }^{*}\right)$,

where $\mathrm{W}^{*}, \mu^{*}, \mathrm{t}_{2}{ }^{*}$ are optimal values of $\mathrm{W}, \mu, \mathrm{t}_{2}$, respectively for case 3 .

\section{NUMERICAL EXPERIMENT AND SENSITIVITY ANALYSIS}

For illustrative purpose, an experiment is performed for each case using MATLAB 7.4.

We consider the values of the parameters in appropriate units such that $\mathrm{a}=2, \mathrm{~b}=0.1, \alpha=0.02, \beta=3.2, \mathrm{~g}=0.4, \mathrm{~h}=0.5, \delta=0.06$, $\mathrm{r}=0.1, \mathrm{c}_{\mathrm{o}}=5, \mathrm{c}_{\mathrm{p}}=1.5, \mathrm{c}_{\mathrm{h}}=0.2, \mathrm{c}_{\mathrm{a}}=0.5, \mathrm{c}_{\mathrm{d}}=0.5, \mathrm{c}_{\mathrm{s}}=1, \mathrm{c}_{1}=2$, $\mathrm{T}=1$.Optimal values of decision variables with minimized total cost with respect to all three cases are given in the form of Table 1 below:

Table 1

\begin{tabular}{c|ccc}
\hline & Case 1 & Case 2 & Case 3 \\
& $\boldsymbol{t}_{1}>\boldsymbol{\mu}$ & $\boldsymbol{t}_{\boldsymbol{1}}<\boldsymbol{\mu}$ & $\boldsymbol{t}_{\boldsymbol{1}}<\boldsymbol{t}_{2}<\boldsymbol{\mu}$ \\
\hline $\boldsymbol{\mu}^{*}$ & 0.0187 & 0.3524 & 1 \\
$\mathbf{t}_{\mathbf{1}}{ }^{*}$ & 0.9754 & 0.208 & 0.39479 \\
$\mathbf{t}_{\mathbf{2}}{ }^{*}$ & 0.93889 & 0.5079 & 0.42545 \\
$\mathbf{W}^{*}$ & 1.39685 & 0.85114 & 0.71243 \\
$\mathbf{Q}_{\mathbf{i}}{ }^{*}$ & 1.5190 & 1.8382 & 14.5445 \\
\hline $\mathbf{T C}_{\mathbf{i}}{ }^{*}$ & 7.3421 & 5.736418 & 3.651939 \\
\hline
\end{tabular}

From the above experimental observations, the optimal solution is obtained from case 3 in which time point $\mu$ occurs after $t_{2}$.

Keeping in view the above experimental results, sensitivity analysis is performed for case 3, which gives optimal solution. It is carried out by changing the parameters by $20 \%$ and $10 \%$ taking one parameter at a time and keeping all other parameters fixed. Change in percentage in total cost of the system, denoted by PCT, corresponding to each value of parameters is calculated. The results of sensitivity analysis are presented in Table 2 and Figure 1. 
Table 2. Sensitivity analysis of various parameters

\begin{tabular}{|c|c|c|c|c|c|c|}
\hline & $\mu$ & $t_{1}$ & $t_{2}$ & $\bar{W}$ & TC & $\overline{P C T}(\%)$ \\
\hline $\mathrm{c}_{\mathrm{o}}+20 \%$ & 0.99999 & 0.39475 & 0.42546 & 0.71245 & 4.652298 & 27.39 \\
\hline $\mathrm{c}_{\mathrm{o}}+10 \%$ & 1 & 0.39479 & 0.42545 & 0.71243 & 4.151940 & 13.69 \\
\hline$c_{o}-10 \%$ & 1 & 0.39486 & 0.42547 & 0.71245 & 3.151964 & -13.69 \\
\hline$c_{o}-20 \%$ & 1 & 0.39486 & 0.42547 & 0.71245 & 2.651964 & -27.38 \\
\hline$c_{p}+20 \%$ & 1 & 0.39479 & 0.42545 & 0.71243 & 3.865667 & 5.85 \\
\hline$c_{p}+10 \%$ & 1 & 0.39479 & 0.42545 & 0.71243 & 3.758803 & 2.93 \\
\hline$c_{p}-10 \%$ & 1 & 0.39479 & 0.42545 & 0.71243 & 3.545076 & -2.93 \\
\hline$c_{p}-20 \%$ & 1 & 0.39465 & 0.42547 & 0.71247 & 3.438221 & -5.85 \\
\hline$c_{h}+20 \%$ & 1 & 0.39468 & 0.42552 & 0.71255 & 3.659103 & 0.19 \\
\hline$c_{h}+10 \%$ & 1 & 0.39479 & 0.42545 & 0.71243 & 3.655335 & 0.09 \\
\hline$c_{h}-10 \%$ & 1 & 0.39479 & 0.42545 & 0.71243 & 3.648554 & -0.09 \\
\hline$c_{h}-20 \%$ & 1 & 0.39479 & 0.42545 & 0.71243 & 3.645149 & -0.19 \\
\hline $\mathrm{c}_{\mathrm{s}}+20 \%$ & 0.99992 & 0.37455 & 0.45211 & 0.75672 & 4.228104 & 15.78 \\
\hline $\mathrm{c}_{\mathrm{s}}+10 \%$ & 0.99951 & 0.39198 & 0.42828 & 0.71713 & 3.887113 & 6.44 \\
\hline $\mathrm{c}_{\mathrm{s}}-10 \%$ & 0.99978 & 0.10972 & 0.28561 & 0.51181 & 2.31286 & -36.67 \\
\hline $\mathrm{c}_{\mathrm{s}}-20 \%$ & 0.99991 & 0.10275 & 0.16255 & 0.29432 & 0.904274 & -75.24 \\
\hline$c_{d}+20 \%$ & 1 & 0.39479 & 0.42545 & 0.71243 & 3.651966 & 0.00 \\
\hline $\mathrm{c}_{\mathrm{d}}+10 \%$ & 1 & 0.39479 & 0.42545 & 0.71243 & 3.651953 & 0.00 \\
\hline$c_{d}-10 \%$ & 1 & 0.39479 & 0.42545 & 0.71243 & 3.651936 & -0.00 \\
\hline$c_{d}-20 \%$ & 1 & 0.39479 & 0.42545 & 0.71243 & 3.651913 & -0.00 \\
\hline $\mathrm{c}_{1}+20 \%$ & 0.99057 & 0.06547 & 0.81891 & 1.51563 & 7.064616 & 93.45 \\
\hline$c_{1}+10 \%$ & 1 & 0.05544 & 0.49355 & 0.91288 & 3.905545 & 6.94 \\
\hline$c_{1}-10 \%$ & 1 & 0.17646 & 0.24608 & 0.43371 & 2.8048067 & -23.20 \\
\hline$c_{1}-20 \%$ & 0.99994 & 0.01031 & 0.01614 & 0.03127 & 1.826674 & -49.98 \\
\hline $\mathrm{c}_{\mathrm{a}}+20 \%$ & 1 & 0.39479 & 0.42545 & 0.71243 & 3.665316 & 0.37 \\
\hline $\mathrm{c}_{\mathrm{a}}+10 \%$ & 1 & 0.39479 & 0.42545 & 0.71243 & 3.658628 & 0.18 \\
\hline$c_{a}-10 \%$ & 1 & 0.39479 & 0.42545 & 0.71243 & 3.645251 & -0.18 \\
\hline$c_{a}-20 \%$ & 1 & 0.39479 & 0.42545 & 0.71243 & 3.638563 & -0.37 \\
\hline
\end{tabular}




\begin{tabular}{|c|c|c|c|c|c|c|}
\hline $\mathrm{b}+20 \%$ & 1 & 0.0995 & 0.1234 & 0.22521 & 0.210123 & -94.25 \\
\hline $\mathrm{b}+10 \%$ & 1 & 0.39048 & 0.43881 & 0.73481 & 3.498597 & -4.20 \\
\hline b- $10 \%$ & 0.99978 & 0.39697 & 0.42872 & 0.71673 & 3.952701 & 8.24 \\
\hline b- $20 \%$ & 0.96962 & 0.27426 & 0.37963 & 0.6477 & 5.337313 & 46.15 \\
\hline$\alpha+20 \%$ & 0.99959 & 0.19368 & 0.27713 & 0.48516 & 2.478569 & -32.13 \\
\hline$\alpha+10 \%$ & 1 & 0.27677 & 0.40384 & 0.68866 & 3.494032 & -4.32 \\
\hline$\alpha-10 \%$ & 0.99943 & 0.38986 & 0.42836 & 0.71739 & 3.701716 & 1.36 \\
\hline$\alpha-20 \%$ & 0.99522 & 0.39026 & 0.42825 & 0.7172 & 3.897945 & 6.74 \\
\hline$\beta+20 \%$ & 0.99995 & 0.38992 & 0.42849 & 0.71751 & 3.678332 & 0.72 \\
\hline$\beta+10 \%$ & 0.99998 & 0.39037 & 0.42841 & 0.71739 & 3.676398 & 0.67 \\
\hline$\beta-10 \%$ & 0.99997 & 0.04087 & 0.30763 & 0.57386 & 2.757020 & -24.51 \\
\hline$\beta-20 \%$ & 1 & 0.00826 & 0.10818 & 0.2094 & 1.005952 & -72.45 \\
\hline $\mathrm{g}+20 \%$ & 1 & 0.16189 & 0.51144 & 0.86965 & 4.350873 & 19.14 \\
\hline $\mathrm{g}+10 \%$ & 0.99974 & 0.29563 & 0.43802 & 0.72903 & 3.761781 & 3.01 \\
\hline g- $10 \%$ & 0.99999 & 0.19383 & 0.31002 & 0.54921 & 2.745481 & -24.82 \\
\hline $\mathrm{g}-20 \%$ & 1 & 0.08479 & 0.19441 & 0.36052 & 1.770064 & -51.53 \\
\hline $\mathrm{h}+20 \%$ & 0.99997 & 0.07136 & 0.0938 & 0.17715 & 0.870086 & -76.17 \\
\hline $\mathrm{h}+10 \%$ & 0.9999 & 0.34744 & 0.39775 & 0.68172 & 3.452088 & -5.47 \\
\hline h- $10 \%$ & 0.9989 & 0.1157 & 0.45974 & 0.8079 & 4.022686 & 10.15 \\
\hline $\mathrm{h}-20 \%$ & 0.99988 & 0.6336 & 0.76192 & 1.17276 & 6.092710 & 66.83 \\
\hline $\mathrm{r}+20 \%$ & 0.99996 & 0.3526 & 0.468 & 0.78462 & 4.556483 & 24.77 \\
\hline $\mathrm{r}+10 \%$ & 0.99954 & 0.40068 & 0.41513 & 0.69561 & 3.928254 & 7.57 \\
\hline $\mathrm{r}-10 \%$ & 0.99989 & 0.23718 & 0.27829 & 0.48371 & 1.942353 & -46.81 \\
\hline r-20\% & 0.99965 & 0.26222 & 0.34049 & 0.58526 & 1.903929 & -47.87 \\
\hline
\end{tabular}

The main observations drawn from above performance are as follows:

1. The percentage change in total cost (PCT) is almost equal for both positive and negative changes in parameters $c_{o}, c_{p}$, $\mathrm{c}_{\mathrm{h}}, \mathrm{c}_{\mathrm{d}}, \mathrm{c}_{\mathrm{a}}$.

2. $\mathrm{PCT}$ is more sensitive for a negative change in percentage of parameters $\beta, g, r, c_{s}$ than an equal positive change in these parameters.
3. PCT is more sensitive for a positive change in parameters $b$, $\alpha, \mathrm{h}, \mathrm{c}_{1}$ than an equal negative change in these parameters.

4. Due to positive and negative change in percentage of parameters $\beta, g, r, h, c_{o}, c_{p}, c_{s}$ and $c_{l}$, PCT increases and decreases respectively but the trend is reversed for the parameters $b, \alpha$ and $h$.

5. Total cost shows negligible sensitivity towards the change in values of parameters $c_{h}, c_{d}$ and $c_{a}$. 

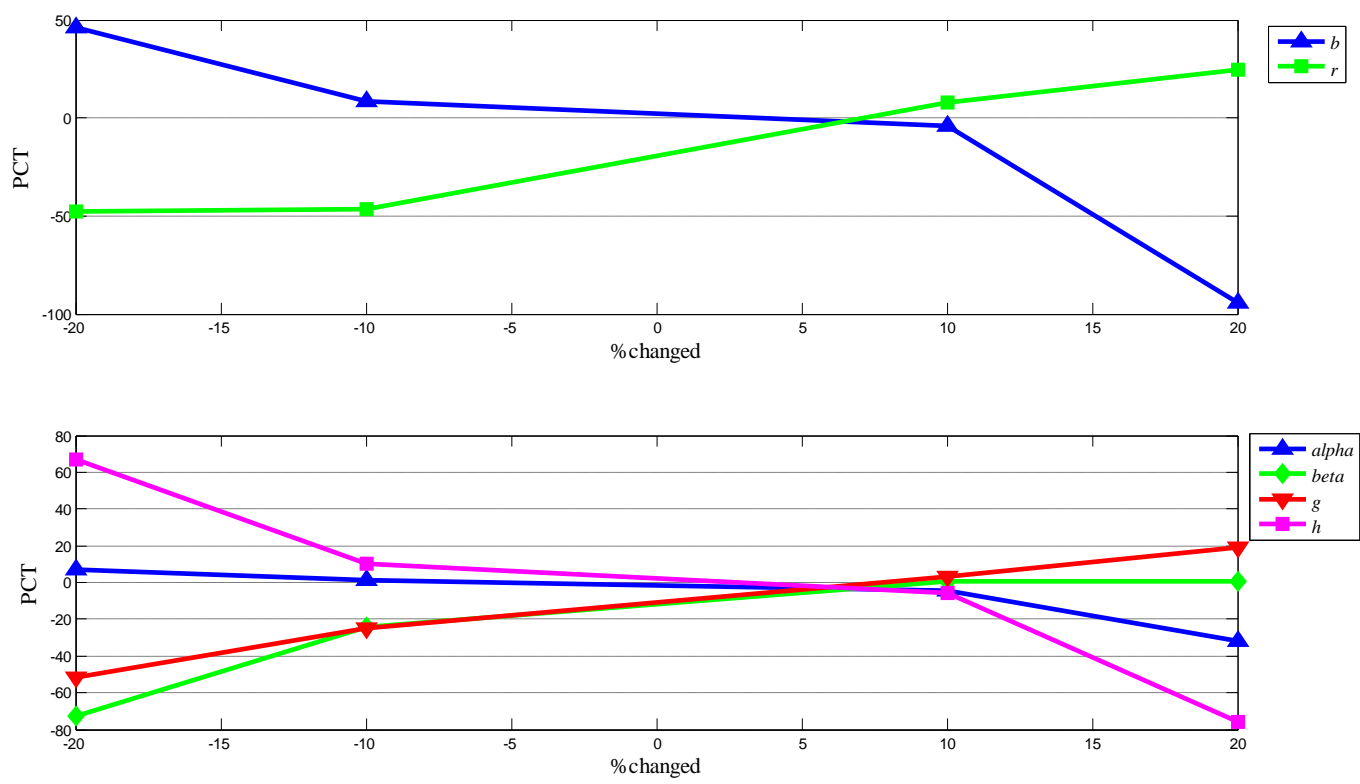

Note: alpha $=\alpha$, beta $=\beta$
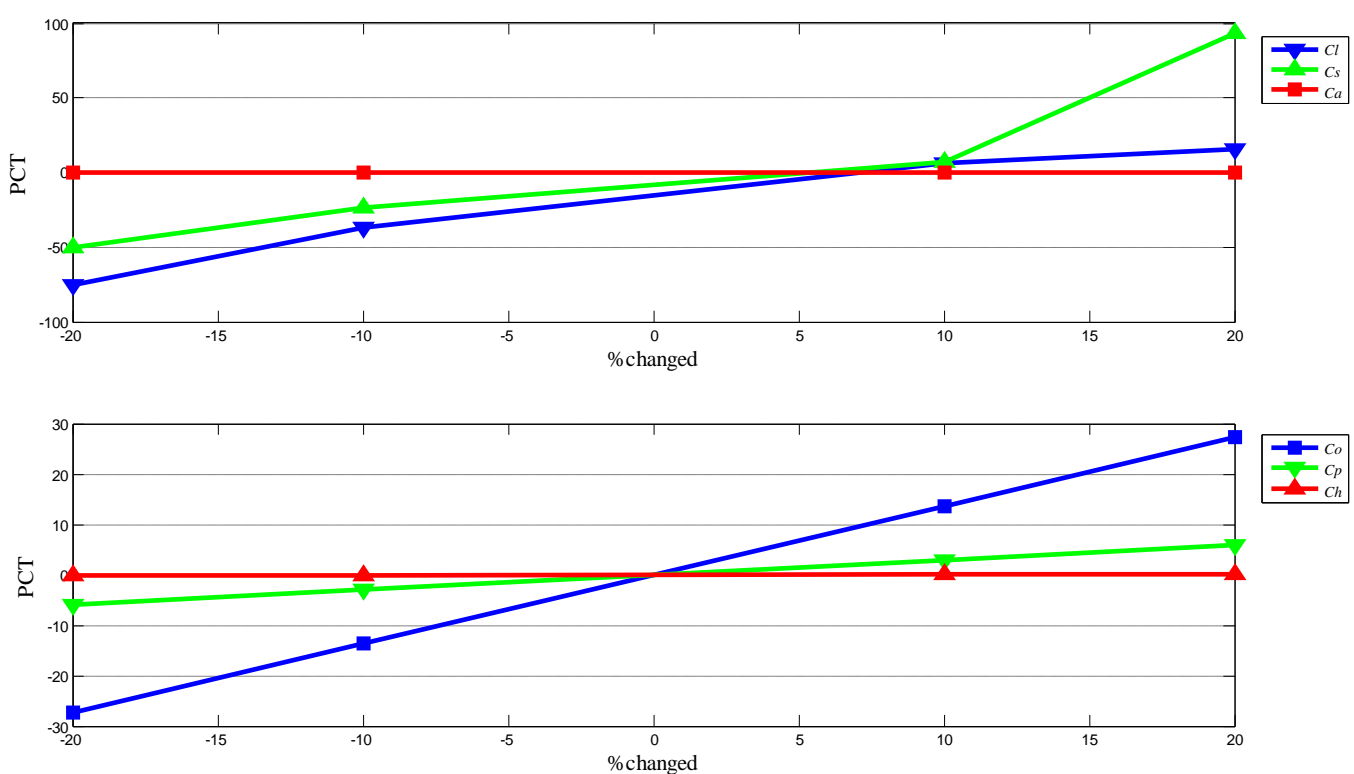

Fig 1: Effects of changing various parameters on total cost

\section{CONCLUDING REMARKS}

For an infinite horizon inventory model that deals with ameliorating/deteriorating items and ramp type pattern of time dependent demand, this paper modifies the solution given by Singh et al. (2011) which suffers from incompleteness. They propose three possible scenarios regarding the demand pattern and derive total cost function for each scenario.

Main objective of the model is to minimize the total relevant cost of the system which includes costs like ordering cost, purchase cost, holding cost, shortage cost, lost sales cost, deterioration and amelioration cost. The optimal strategy is one which fulfills model's objective. The observations are made with the help of numerical example and the impact of various factors on the total relevant cost is studied by means of sensitivity analysis. It is found that the total cost is minimum when demand of items stabilizes somewhere within the period of shortages. The observations show that the component of demand, denoted by $b$, is one of the important factors, so it must be chosen carefully. In this work, the rates of deterioration and amelioration of items both are taken time varying and follow two parameter Weibull distribution. The study of impact of its parameters on the system indicates their significant role. Therefore, one must choose these parameters accordingly. Further analysis shows that lower the costs such as ordering cost, purchase cost, holding cost, shortage cost, lost sales cost, deterioration and amelioration cost, lower will be the total relevant cost which is again true in real life. Also, as we reduce the factor representing the difference between inflation and time value of money, total cost of the system reduces. It concludes that in order to obtain lesser total cost, the rate of inflation must slow down .The analysis helps us 
formulate a generic model for further work. Hence, all the efforts have been carefully directed towards the possible futuristic enhancements of the model.

\section{REFERENCES}

[1] Deng, P.S., Lin, Robert H.-J. and Chu, P. 2007. A note on the inventory models for deteriorating items with ramp type demand rate. European Journal of Operational Research 178, 112-120.

[2] Giri, B.C., Jalan, A.K. and Chaudhuri, K.S. 2003. Economic order quantity model with Weibull deterioration distribution, shortage and ramp-type demand. International Journal of Systems Science, 34, 237-243.

[3] Hill, R.M. 1995. Inventory model for increasing demand followed by level demand. Journal of the Operational Research Society, 46, 1250-1259.

[4] Mandal, B. and Pal, A. K. 1998. Order level inventory system with ramp type demand for deteriorating items. Journal of Interdisciplinary Mathematics, 1, 49-66.

[5] Mandal, B. 2010. An EOQ model with Weibull distributed deteriorating items under ramp type demand and shortages. Opsearch, 47, 158-165.

[6] Manna, S.K. and Chiang, C. 2010. Economic production quantity models for deteriorating items with ramp type demand. International Journal of Operational Research, 7, 429-444.

[7] Panda, S., Senapati, S. and Basu, M. 2008. Optimal replenishment policy for perishable seasonal products in a season with ramp-type time dependent demand. Computers \& Industrial Engineering, 54, 301-314.

[8] Roy, T. and Chaudhuri, K.S. 2011. A finite time horizon EOQ model with ramp-type demand rate under inflation and time-discounting. International Journal of Operational Research, 11, 100-118.

[9] Singh, S.R., Kumar, T. and Gupta, C.B. 2011. Optimal replenishment policy for ameliorating item with shortages under inflation and time value of money using genetic algorithm. International Journal of Computer Applications, 27, 5-17.

[10] Skouri, K. , Konstantaras, I., Papachristos, S. and Ganas, I. 2009. Inventory models with ramp type demand rate, partial backlogging and Weibull deterioration rate. European Journal of Operational Research, 192, 79-92.

[11] Wu, J.W., Lin, C., Tan, B. and Lee, W.C. 1999. An EOQ model with ramp type demand rate for items with Weibull deterioration. International Journal of Information and Management Sciences, 10, 41-51. 